\title{
Validation of a portable, non-mydriatic fundus camera compared to gold standard dilated fundus examination using slit lamp biomicroscopy for assessing the optic disc for glaucoma
}

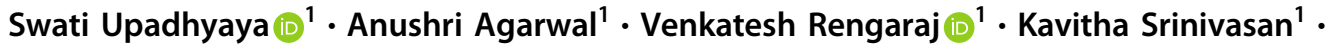 \\ Paula Anne Newman Casey $\mathbb{1}^{2} \cdot$ Emily Schehlein ${ }^{2}$
}

Received: 15 August 2020 / Revised: 28 January 2021 / Accepted: 19 February 2021 / Published online: 11 March 2021

(c) The Author(s), under exclusive licence to The Royal College of Ophthalmologists 2021

\begin{abstract}
Purpose To evaluate the sensitivity and specificity of a portable non-mydriatic fundus camera to assess the optic disc for glaucoma.

Methods We conducted a single-site, cross-sectional, observational, instrument validation study. Non-mydriatic fundus photographs centred at the optic disc were obtained from 276 eyes of 68 glaucoma and 70 normal patients, using a portable fundus camera (Smartscope, Optomed, Oulu, Finland). A senior Glaucoma consultant, masked to the patient's study participation, performed a gold standard dilated fundus examination to make the diagnosis of glaucoma. Following this, a mydriatic photograph was taken by a standard table-top fundus camera. All the images were digitalized and de-identified by an independent investigator and presented to two remote graders, masked to the patients, their diagnoses, and photographic modality. Based on individual disc characteristics, a diagnosis of screening positive or negative for glaucoma was made. In the end, the independent investigator re-identified the images. Sensitivity and specificity to detect glaucoma with the undilated Smartscope camera was calculated compared to dilated fundus examination.

Results Grading remote images taken with the portable non-mydriatic fundus camera showed a sensitivity of $96.3 \%(95 \%$ confidence interval (CI): 91.6-98.8\%) and $94.8 \%$ (95\% CI: 89.7-97.9\%) and a specificity of $98.5 \%$ (95\% CI: 94.9-99.8\%) and $97.8 \%$ (95\% CI: 93.9-99.6\%) for the two graders respectively as compared to gold standard dilated fundus examination. Conclusion The non-mydriatic Smartscope fundus images have high sensitivity and specificity for diagnosing glaucoma remotely and thus may be an effective tool for use in community outreach programs.
\end{abstract}

\section{Introduction}

Globally, at least 1 billion people have vision impairment. This includes those with vision impairment or blindness due to unaddressed refractive error and presbyopia (949 million), cataract (65.2 million), glaucoma (6.9 million),

Supplementary information The online version contains supplementary material available at https://doi.org/10.1038/s41433021-01485-2.

Swati Upadhyaya

swati.dr@aravind.org

1 Aravind Eye Hospital and Post Graduate Institute of Ophthalmology, Pondicherry, India

2 Kellogg Eye Center, University of Michigan, Ann Arbor, MI, USA corneal opacities (4.2 million), diabetic retinopathy (3 million), and trachoma (2 million) [1].

The number of individuals with glaucoma worldwide is projected to increase to 112 million in 2040 [2]. Approximately $50 \%$ of those with glaucoma remain undiagnosed in the United States (US) and in the developing world, the prevalence of known disease is even lower [3], with rates of undiagnosed glaucoma of up to $90 \%$ in rural India [4]. Many glaucoma patients are diagnosed only in the advanced stages when there is a low probability of being able to preserve usable vision [5-7]. Thus, early detection is crucial to initiate treatment in the earliest phases of glaucoma and to avoid its natural progression to blindness.

Glaucoma has been described as an ideal disorder for screening as it is an asymptomatic condition with an extended course before visual impairment occurs [8]. Screening strategies that rely only on IOP measures and that neglect disc and visual field assessment are inadequate [9]. 
The current "gold standards" for glaucoma diagnosis are optic disc assessment (monitors structural change) and standard achromatic white on white perimetry (monitors functional change) [10]. Optic discs can be evaluated using various tools including direct ophthalmoscopy and slit lamp biomicroscopy. Both of these examination tools require close proximity between the patient and the examiner. This proximity is not always possible-particularity in rural areas that may not have access to an ophthalmologist or in instances of disease, such as the COVID-19 pandemic. An alternative method to assess the structure of the optic disc is fundus photography, which have traditionally utilized large table-top fundus cameras. These cameras require pupillary dilatation and are costly and are not portable. Likewise, assessing perimetry in the community using traditional visual field analysers, such as a Humphrey or Octopus are difficult to use because the machines are non-portable and costly. Newer portable virtual reality-based perimeters may simplify screening at remote locations in the future [11]. Similarly, using a portable non-mydriatic fundus camera may be a reasonable way to improve access to high-quality glaucoma screening in community outreach camps where screening is done with limited resources and dilating every patient is not possible.

Continued research into how to improve both the accuracy and cost effectiveness of glaucoma screening is imperative. According to US Preventive Services Task Force recommendation, the cost effectiveness of screening for glaucoma varies widely depending on the cost of the tests used [12]. Recent studies have demonstrated that the modality of screening is also important as community-based glaucoma screening in rural and urban India are more costeffective compared to opportunistic screening [13, 14].

Most previously tested cameras are table-top models, which have limited portability and applicability in rural outreach settings, especially in the developing world. A study in Nepal compared the cup to disc ratio (CDR) measurements from images taken with a portable nonmydriatic fundus camera to images from a standard, nonportable, mydriatic fundus camera and found reasonable agreeement [15]. Our goal is to validate the use of fundus photographs taken with a non-mydriatic portable fundus camera as compared to the current gold standard in glaucoma diagnosis: dilated fundus examination and visual field evaluation.

The purpose of this study is: (1) to compare the CDR measurement between non-mydriatic fundus photograph (NMFP) assessment and dilated fundus examination using slit lamp biomicroscopy and (2) to compare the diagnosis of glaucoma between remote graders using only images from a portable, non-mydriatic camera and an in-person gold standard dilated fundus examination (DFE) using slit lamp biomicroscopy.

\section{Material and methods}

A single-site, cross-sectional, observational, instrument validation study was conducted at the Aravind Eye Hospital (AEH), Pondicherry, India, from August 2015 to June 2017. The study was approved by the Institutional Ethics Committee of AEH and adhered to the tenets of the Declaration of Helsinki. Written informed consent was obtained from all the participants. Consent to publish patients' photographs with the device in use was obtained.

\section{Participants}

Two groups of participants, aged 30 to $\leq 70$ years, were recruited: (1) clinically proven glaucoma patients with typical optic nerve head (ONH) and visual field changes presenting to glaucoma services for review check-up and (2) patients presenting to comprehensive clinics with no clinical evidence of glaucoma (control group).

Diagnosed cases of Primary open-angle glaucoma (POAG), primary angle-closure glaucoma (PACG), and normal-tension glaucoma (NTG) with previous reliable visual field test (Humphrey Field Analyser 24-2 Swedish Interactive Threshold Algorithm (SITA) Standard) were included in the study. For controls, participants with IOP $<20 \mathrm{mmHg}$, no evidence of glaucoma, no family history of glaucoma, a CDR of $\leq 0.5$, inter-eye asymmetry of $\leq 0.2$, myopia or hyperopia $\leq 3 \mathrm{D}$ were invited to participate in the study.

Participants were excluded if they had any secondary glaucoma, cataract grade $\geq$ Nuclear colour 3, Nuclear Opalescence 3, Cortical cataract 3, or posterior subcapsular cataract 3 (P3) as per the Lens Opacification Classification System III, and/ or posterior capsular opacification so that media clarity did not limit optic disc assessment both on slit lamp biomicroscopy and fundus imaging. Vitreoretinopathy of any aetiology except mild to moderate nonproliferative diabetic retinopathy, and any optic neuropathy other than glaucoma were also excluded.

\section{Clinical assessment and photography}

All study participants underwent an extensive ophthalmologic evaluation including Snellen best-corrected visual acuity, slit lamp examination, intraocular pressure (IOP) measurement with Goldmann applanation tonometry, and gonioscopy using a Goldman two mirror gonio lens (Volk Optical). Visual fields were performed on a Humphrey perimeter (HFA II, Carl Zeiss Meditec, Dublin, California, USA) with the SITA Standard 24-2 strategy only for diagnosed glaucoma patients as per their review protocol. A reliable visual field was defined as one with fixation losses, false negatives and false positives $\leq 20 \%$. 
Fig. 1 Non-mydriatic Fundus Camera. a Smartscope in Use. b Image on Smartscope.

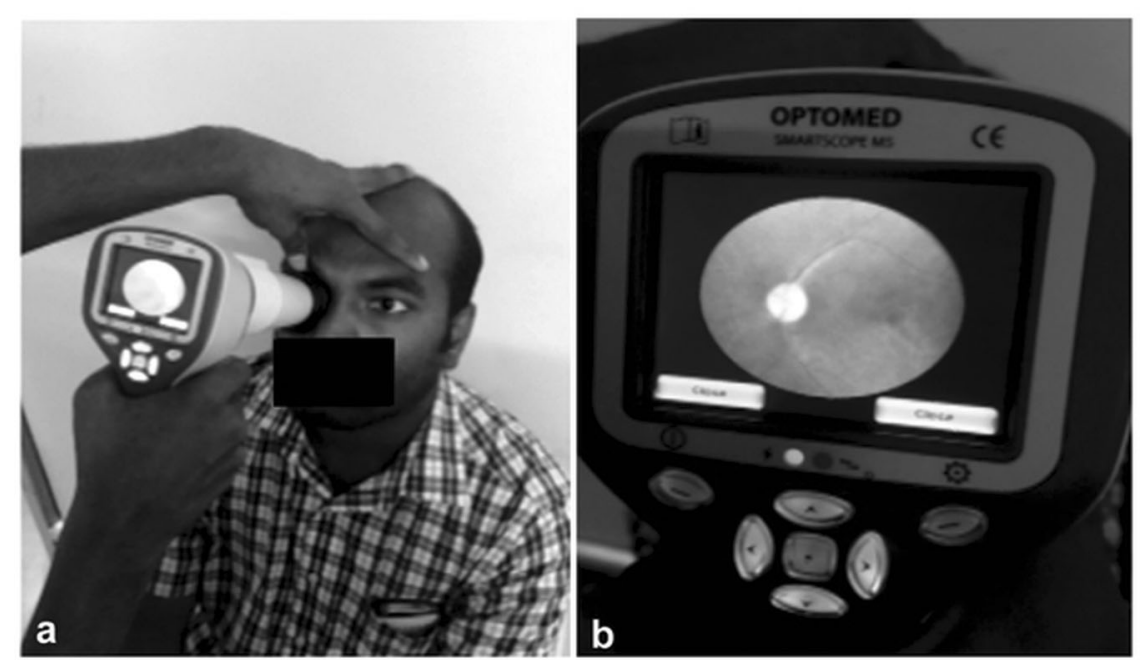

Each eye had undilated coloured image centred on the optic disc taken using Smartscope non-mydriatic fundus camera (Optomed M5, Oulu, Finland) in a dimly illuminated room.

A single-trained ophthalmic photographer took all the images (Fig. 1, Supplementary Figs. 1a, b and 2a, b). The photographer was trained in taking images by both Smartscope $\left(45^{\circ}\right)$ and standard table-top $\left(50^{\circ}\right)$ fundus camera (Topcon, Tokyo, Japan) and had done similar photography for a previous study of diabetic retinopathy screening that included 3000 images [16].

The Smartscope is a handheld, non-mydriatic $\left(45^{\circ}\right)$ digital fundus camera that provides general, ophthalmoscopic, otoscopic and dermatoscopic imaging with a fivemegapixel image sensor. It weighs $400 \mathrm{~g}$ ( 0.88 pounds) and is powered with a battery which gives the freedom to move around and take the device to any location. The Smartscope has autofocus capability and a built-in LED light source, is $\mathrm{Wi}-\mathrm{Fi}$ enabled, and produces an image resolution of $2560 \times$ 1920 pixels, which was compressed to $1280 \times 960$ pixels post transmission.

The pupils were then dilated, and a senior glaucoma specialist (RV) with more than 15 years of experience, who was masked to patient's participation in the study, performed the DFE using slit lamp biomicroscopy with a $90 \mathrm{D}$ lens (Volk Optical). The following $\mathrm{ONH}$ findings were assessed:

(1) Vertical cup to disc ratio (VCDR)

(2) Neuroretinal rim (NRR) appearance-notch, thinning, loss

(3) Retinal nerve fibre layer defect (RNFLD)—present or absent

(4) Baring of circumlinear vessels (BCLV)—present or absent
(5) Disc haemorrhage_-present or absent

(6) Peri-papillary atrophy_present or absent

Glaucoma was defined as the optic disc showing characteristic glaucomatous changes in terms of VCDR $(>0.6)$ with or without NRR notch/loss, BCLV, RNFLD, or PPA on dilated fundus exam, with a corresponding visual field defect.

Based on this comprehensive work up, a final diagnosis of glaucoma or normal was made. This was used as the gold standard for comparing the diagnosis made on Smartscope imaging.

Following this, dilated fundus imaging was performed with the Smartscope and a standard table-top $\left(50^{\circ}\right)$ fundus camera TRC-50DX (Topcon, Tokyo, Japan) (Supplementary Figs. 1c, d and 2c, d). The Topcon camera has an attached Canon SLR camera that produced an image resolution of $4200 \times 2800$ pixels post transmission.

The definition of screening positive for glaucoma on Smartscope imaging was the $\mathrm{ONH}$ showing characteristic glaucomatous changes in terms of VCDR $(>0.6)$ with or without NRR notch/ loss, BCLV, RNFLD, or PPA.

\section{Remote interpretation of fundus photographs}

All the images stored as Joint Photographic Experts Group files were digitalized, de-identified, randomized, and given a unique identification number using a random number generator by an independent investigator (AA). The images were presented separately to two masked glaucoma specialists with more than ten years of experience in glaucoma at the AEH (SK and US). Both graders used a designated computer screen which was $\geq 17$ diagonal inches. The resolution of the screen was $\geq 1600 \times 2000$ pixels. We used standard brightness and contrast settings set by 
Windows 10. Graders were not allowed to manipulate the images. Randomly selected images were presented to the graders in sets of 60 in a single sitting.

VCDR measurements and the diagnosis of glaucoma made from non-dilated images from the Smartscope were compared with standard DFE and dilated Topcon images. Intraobserver reliability was assessed.

\section{Sample size calculation}

The primary outcome measure for the study was the sensitivity and specificity of detecting glaucoma using nonmydriatic Smartscope images compared to standard DFE using slit lamp biomicroscopy. Based on the studies by Yogesan et al. [17] and Munoz-Negrete et al. [18], we estimated that 276 eyes from 138 subjects would provide $80 \%$ power to detect a sensitivity of $90 \%$ for the classification of eyes as glaucomatous by non-mydriatic, portable camera compared to dilated fundus exam, assuming an alpha of 0.05 .

\section{Statistical analysis}

Participant characteristics were summarized using means to quantify continuous variables and frequencies and percentages to quantify categorical variables. Student's $t$ test was used to compare differences between continuous variables and Chi square test was used to compare differences between categorical variables. Interclass correlation coefficients were calculated to compare the CDR grading and kappa statistics were used to compare the classification of glaucoma based on the two masked graders' evaluation of the fundus photographs.

Bland-Altman plots were used to compare the agreement in CDR grading between the masked graders. STATA 11.1 (Texas, USA) was used to conduct the statistical analyses. $P$ values of less than 0.05 were considered statistically significant.

\section{Results}

\section{Demographic characteristics of the study population}

A total of 173 participants were approached and 148 agreed to participate (86\% response rate). 138 participants (276 eyes) were included in the analysis as ten participants (20 eyes) were excluded due to missing photographs of either eye. The mean age of glaucoma patients $(n=68)$ was significantly higher $(59.97 \pm 8.86$ years (Mean \pm SD) $)$ compared to the controls $(n=70)(47.88 \pm 8.36$ years). Overall, $37 \%$ of the study participants were female, with no significant difference in gender between the groups $(p=0.95)$.

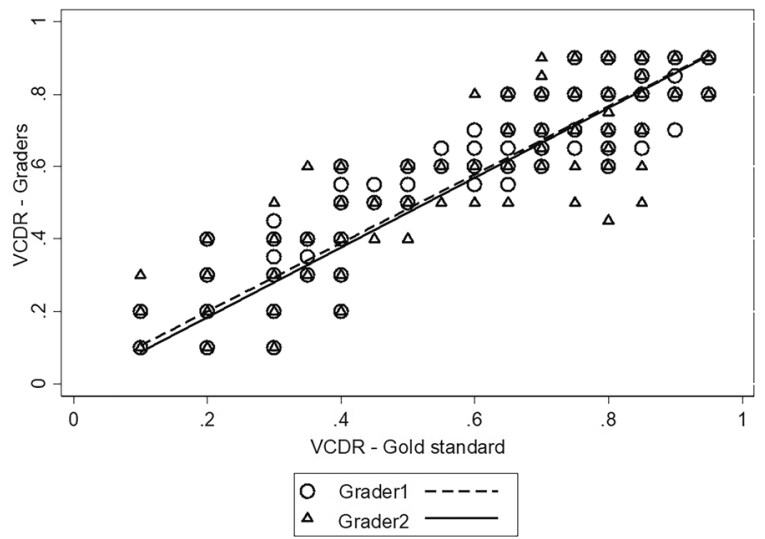

Fig. 2 Scatter plot for VCDR by grader 1 and grader 2 as compared to gold standard dilated fundus exam.

In the glaucoma group, 45 participants had POAG, 15 had PACG and 8 had NTG. The average VCDR in the glaucoma group was 0.7 and in the control group was 0.3.

\section{Vertical CD ratio (VCDR)}

Intra class correlation (ICC) was measured using a two-way mixed effects model which measures the consistency of agreement of vertical $\mathrm{CD}$ ratio between the gold standard (DFE-dilated fundus exam) and undilated Smartscope by grader 1 (ICC $=0.98 ; 95 \%$ CI $0.97-0.98)$ and grader 2 (ICC $=0.94$; 95\% CI 0.92-0.95) (Supplementary Fig. 3) and among the two graders ( $\mathrm{ICC}=0.95$; 95\% CI 0.94-0.96). There was a high degree of reliability in grading VCDR for both graders on images acquired with the undilated Smartscope as shown in the scatter plot in Fig. 2.

\section{Interobserver variability}

A Bland-Altman plot was used to visualize and assess the differences in measuring the VCDR between the two graders for the undilated Smartscope images. The mean difference with the undilated Smartscope images was 0.010 CDR amount, with the limits of agreement being -0.14 and +0.16 (Fig. 3).

\section{Glaucoma diagnosis}

There was strong agreement for the diagnosis of glaucoma between the two remote graders using the non-dilated fundus images $(k=0.95$; 95\% CI $0.91-0.99)$.

\section{Sensitivity and specificity to detect glaucoma}

The sensitivity of the undilated Smartscope images as compared to the DFE for the two graders was found to be $96.3 \%$ 


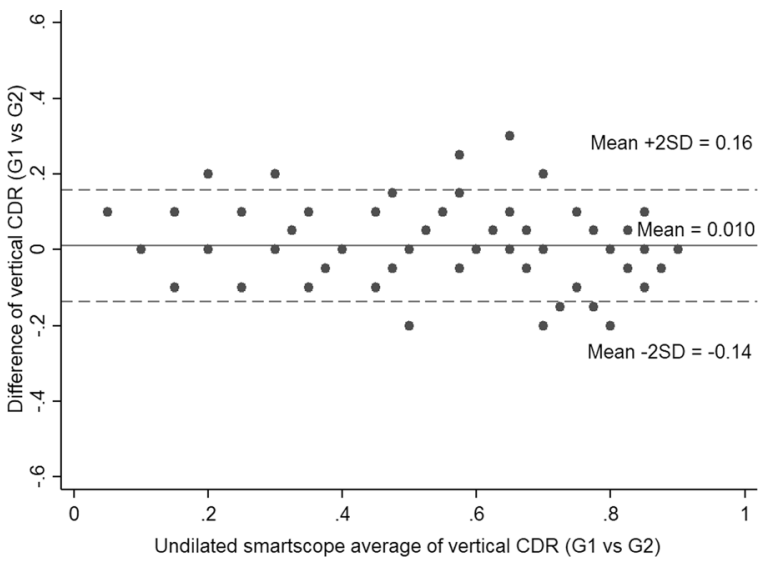

Fig. 3 Bland-Altman plot showing difference in VCDR between the two graders on undilated Smartscope images.

Table 1 Sensitivity and specificity of undilated Smartscope vs. dilated fundus exam (DFE).

\begin{tabular}{llll}
\hline Undilated Smartscope vs. DFE $(n=$ eyes $)$ & \\
\hline $\begin{array}{llll}\text { Undilated } \\
\text { Smartscope G1 }\end{array}$ & DFE G1 & $\begin{array}{l}\text { Sensitivity and } \\
\text { specificity }(\mathrm{CI})\end{array}$ \\
\cline { 2 - 4 } SP glaucoma & No glaucoma & \\
SN glaucoma & 131 & 2 & $\begin{array}{l}\text { Sensitivity: } 96.32 \% \\
(91.6-98.8 \%)\end{array}$ \\
Total & 5 & 138 & $\begin{array}{l}\text { Specificity: 98.57\% } \\
(94.9-99.8 \%)\end{array}$ \\
\hline $\begin{array}{l}\text { Undilated } \\
\text { Smartscope G2 }\end{array}$ & DFE G2 & 140 & $\begin{array}{l}\text { Sensitivity and } \\
\text { specificity }(\mathrm{CI})\end{array}$ \\
\hline Slaucoma & No glaucoma & \\
\hline SP glaucoma & 129 & 3 & $\begin{array}{l}\text { Sensitivity: 94.85\% } \\
(89.7-97.9 \%)\end{array}$ \\
SN glaucoma & 7 & 137 & $\begin{array}{l}\text { Specificity: 97.86\% } \\
(93.9-99.6 \%)\end{array}$ \\
Total & 136 & 140 & \\
\hline
\end{tabular}

$S P$ screening positive, $S N$ screening negative, $G$ grader.

(95\% CI: 91.6-98.8) and 94.8\% (95\% CI: 89.7-97.9) respectively and the specificity was $98.5 \%(94.9-99.8 \%)$ and 97.8\% (95\% CI: 93.9-99.6), respectively (Table 1).

The sensitivity of the undilated Smartscope images as compared to the dilated fundus images was found to be $97.7 \%$ (95\% CI: 93.4-99.5) and 95.5\% (95\% CI: 90.5-98.3) and specificity was $96.5 \%(92.1-98.9 \%)$ and $97.1 \%$ (95\% CI: 92.9-99.2), respectively, for grader 1 and 2 (Table 2).

\section{Discussion}

Our study using non-mydriatic Smartscope fundus images shows a sensitivity and specificity of $>90 \%$ to detect glaucoma as compared to gold standard DFE. In developing
Table 2 Sensitivity and specificity of undilated Smartscope vs. dilated Topcon.

\begin{tabular}{|c|c|c|c|}
\hline \multicolumn{4}{|c|}{ Undilated Smartscope vs. dilated Topcon } \\
\hline \multirow{2}{*}{$\begin{array}{l}\text { Undilated } \\
\text { Smartscope G1 }\end{array}$} & \multicolumn{2}{|c|}{ Dilated Topcon-G1 } & \multirow{2}{*}{$\begin{array}{l}\text { Sensitivity and } \\
\text { specificity (CI) }\end{array}$} \\
\hline & Glaucoma & No glaucoma & \\
\hline SP Glaucoma & 128 & 5 & $\begin{array}{l}\text { Sensitivity: } 97.71 \% \\
(93.4-99.5 \%)\end{array}$ \\
\hline SN Glaucoma & 3 & 140 & $\begin{array}{l}\text { Specificity: } 96.55 \% \\
(92.1-98.9 \%)\end{array}$ \\
\hline Total & 131 & 145 & \\
\hline \multirow{2}{*}{$\begin{array}{l}\text { Undilated } \\
\text { Smartscope G2 }\end{array}$} & \multicolumn{2}{|l|}{ Topcon-G2 } & \multirow{2}{*}{$\begin{array}{l}\text { Sensitivity and } \\
\text { specificity (CI) }\end{array}$} \\
\hline & Glaucoma & No glaucoma & \\
\hline SP glaucoma & 128 & 4 & $\begin{array}{l}\text { Sensitivity: } 95.52 \% \\
(90.5-98.3 \%)\end{array}$ \\
\hline SN glaucoma & 6 & 138 & $\begin{array}{l}\text { Specificity: } 97.18 \% \\
(92.9-99.2 \%)\end{array}$ \\
\hline Total & 134 & 142 & \\
\hline
\end{tabular}

$S P$ screening positive, $S N$ screening negative, $G$ grader.

countries, where eye care services are often limited and many glaucoma patients present with end-stage disease or blindness in at least one eye, enacting community eye outreach programs may help implement earlier diagnosis and management to prevent blindness from the disease $[14,15]$. Of note, the fact that this is a highly specific test will reduce the numbers of false-positive referrals, which represent a large burden for patients traveling from far away.

Our study brought a range of new techniques to the evaluation of how well non-dilated monoscopic optic disc images can aid in making a diagnosis of glaucoma. A study done at the Tilganga Institute of Ophthalmology (Kathmandu, Nepal) [15] compared the VCDR measurements from images taken with a portable non-mydriatic fundus camera (Pictor, Volk) to images from a standard, non-portable, mydriatic fundus camera (Topcon) and only moderate agreement was found between graders. In this study, images graded by the remote graders had a high level of between grader agreement (ICC 0.98 and 0.94 ). This may be due to the fact that both remote graders were trained in the same institute and had equal amount of experience in the field of glaucoma. Our study compared undilated Smartscope with dilated Topcon fundus photographs in addition to the primary objective of comparing undilated Smartscope images with gold standard DFE. We found a sensitivity and specificity of $97.7 \%$ and $96.5 \%$ respectively for grader 1 and $95.5 \%$ and $97 \%$ respectively for grader 2 . This is in contrast to $41.2-59.0 \%$ sensitivity in previous work comparing the undilated Smartscope images to dilated images obtained on a non-portable table-top camera. In a study conducted by Yogesan et al. [17], the gold standard used for measuring 
VCDR was monoscopic photographic slides whereas in our study DFE using slit lamp biomicroscopy was used as the gold standard. In our study, remote graders used only disc photographs for assessing whether a patient had glaucoma or not whereas in the study by Detry-Morel et al. [19] NMFP and visual field testing by frequency doubling perimeter were used for glaucoma screening. Sensitivity and specificity of undilated disc photographs to identify glaucoma was found to be $58.6 \%$ and $64.3 \%$ respectively whereas our study shows a sensitivity and specificity of $>90 \%$ for photographs alone without perimetry with nonmydriatic photographs.

Our study used only digitalized coloured fundus photographs while Tuulonen et al. [20] used non-mydriatic black and white paper prints obtained from a non-portable Canon retinal camera, focusing on retinal nerve fibre layer defects and did not take IOP into consideration for screening. In our study also, the assessment of the photographs for glaucoma did not include an assessment of IOP, since tonometry was not a part of the protocol. However, we do recommend a future study where red-free and colour photographs both should be compared and taken into consideration as retinal nerve fibre layer defects may be easier to evaluate on red-free imaging.

In a similar study conducted by Sengupta et al. [16], using the same undilated Smartscope to detect visionthreatening diabetic retinopathy (VTDR), they found a sensitivity of $93 \%$ and $88 \%$ and a specificity of $84 \%$ and $90 \%$ between the two masked graders respectively, as compared to DFE, showing that this camera has high reliability in detecting VTDR. [18] Given that this camera has high sensitivity and specificity for imaging the retina both for glaucoma and VTDR, it may be a very important tool in bringing improved screening of the posterior pole to community outreach programs and also in primary eye care centres managed by optometrists or mid-level ophthalmic personnel. These images can be shared with the base hospital for expert opinion thus facilitating referral for further evaluation. Furthermore, these images can be utilized for developing Artificial Intelligence algorithms and take glaucoma screening and diagnosis to the next level.

This study has several limitations. Our study population did not include glaucoma suspects or subjects with mild glaucoma with normal visual fields and also patients with media opacities and so the data do not generalize to those populations. It also may be more difficult to assess cup-todisc ratios in the middle range and make an accurate diagnosis of glaucoma without additional information, such as visual field results, IOP, family history and OCT, which may account for the high sensitivities and specificities reported in this study as compared to previous literature which included a larger range of VCDR. As this study was designed to validate an instrument, only diagnosed cases of glaucoma and participants with normal non-glaucomatous optic discs were included and Humphrey visual fields were not performed for the control group.

All the photographs were taken by a single technician who was trained for a previous study on diabetic retinopathy [16]. This can be both a limitation and a strength. While it is a strength to have high-quality images for purposes of this validation study to assess the instrument's capabilities, it is a limitation to implementation in that this portable fundus camera requires sufficient training and a high enough volume of patients to attain and maintain skill levels [21]. If a health care worker in a remote setting did not have substantial daily practice taking photographs, the quality of the images may be diminished.

This study has several strengths. A single ophthalmologist who was masked to patients' participation performed the gold standard DFE for all the patients and controls included in the study. The remote graders were masked to the camera details as well as to the participants' diagnosis. The images were handled by an independent investigator who was neither involved in patient examination nor grading of images, adding validity to our study.

A portable, non-mydriatic fundus camera could be a useful tool in glaucoma screening in a number of different capacities. At the Aravind Eye Care System, small vision centres run by ophthalmic technicians are connected to the main hospital and ophthalmic consultation via telemedicinebased video conferencing. In addition, the portable nonmydriatic fundus cameras could be used in eye camps to screen for glaucoma, diabetic retinopathy and other pathology in the posterior pole without necessitating dilation. In a tertiary care setting, the portable fundus cameras could be used in place of expensive table-top fundus cameras for recording the glaucomatous changes and its progression on follow-ups. The Smartscope weighs $400 \mathrm{~g}(0.88$ pounds) and costs approximately US $\$ 4000$ whereas Topcon system weighs $\sim 35 \mathrm{~kg}$ (77 pounds) and costs approximately US $\$ 10,000$.

Further studies should evaluate the accuracy of imaging using portable, non-mydriatic camera in a community outreach setting with all ranges of VCDRs, glaucoma suspects and patients with media opacities, to test the potential of the instrument in identifying glaucoma. Future studies should also evaluate whether including red-free images from a nonmydriatic fundus camera might increase the sensitivity of glaucoma diagnosis from photographs alone in comparison to a gold standard clinical evaluation.

We conclude that photographs taken by a non-mydriatic handheld Smartscope fundus camera are comparably effective in detecting glaucoma as compared to gold standard DFE as well as photographs taken by table-top fundus camera and it has the potential of becoming a reliable tool both for community outreach as well as telemedicine.

Supplementary material is available on Eye's website. 


\section{Summary}

\section{What is known about this topic}

- Non-mydriatis fundus camera have high sensitivity and specificity to detect vision threatening diabetic retinopathy.

- Taking images with non-mydriatic fundus camera, has a learning curve.

\section{What this study adds}

- Non-mydriatic fundus camera has high sensitivity and specificity to detect glaucoma.

- Non-mydriatic cameras can be helpful in community outreach programs to diagnose glaucoma.

- Training a single technician in taking images with nonmydriatic cameras, greatly increasesthe quality of images.

Acknowledgements We would like to acknowledge the valuable contributions of Mr. Saravanan Sathish (Ophthalmic photographer) and Bio-statistician Mr. Mohammed Sithiq to this work.

Author contributions US designed the study. AA made the study proforma, recruited study participants, collected all the images, deidentified the images, and made sets to be presented to masked graders (SK and US). RV performed the gold standard fundus examination. US wrote the manuscript with contributions from NCPA, SE, and SK.

\section{Compliance with ethical standards}

Conflict of interest The authors declare no competing interests.

Publisher's note Springer Nature remains neutral with regard to jurisdictional claims in published maps and institutional affiliations.

\section{References}

1. Bourne RRA, Flaxman SR, Braithwaite T, Cicinelli M, Das A, Jonas JB. Vision Loss Expert Group. Magnitude, temporal trends, and projections of the global prevalence of blindness and distance and near vision impairment: a systematic review and metaanalysis. Lancet Glob Health. 2017;5:e888-97.

2. Quigley HA, Broman AT. The number of people with glaucoma worldwide in 2010 and 2020. Br J Ophthalmol. 2006;90:262-7.

3. Tham YC, Li X, Wong TY, Quigley HA, Aung T, Cheng C. Global prevalence of glaucoma and projections of glaucoma burden through 2040: a systematic review and meta-analysis. Ophthalmology. 2014;121:2081-90.
4. Lee BW, Sathyan P, John RK, Singh K, Robin AL. Predictors of and barriers associated with poor follow-up in patients with glaucoma in south India. Arch Ophthalmol. 2008;126:1448.

5. Chen PP. Blindness in patients with treated open-angle glaucoma. Ophthalmology. 2003;110:726-33.

6. Wilson R, Walker AM, Dueker DK, Crick RP. Risk factors for rate of progression of glaucomatous visual field loss: a computerbased analysis. Arch Ophthalmol. 1982;100:737-41.

7. Grant WM, Burke JF. Why do some people go blind from glaucoma? Ophthalmology. 1982;89:991-8.

8. Quigley HA. Current and future approaches to glaucoma screening. J Glaucoma. 1998;7:210-20.

9. Sommer A. Epidemiology as it relates to screening for glaucoma. Surv Ophthalmol. 1989;33:441-2.

10. Medeiros FA, Zangwill LM, Bowd C, Sample PA, Weinreb RN. Use of progressive glaucomatous optic disk change as the reference standard for evaluation of diagnostic tests in glaucoma. Am J Ophthalmol. 2005;139:1010-8. https://doi.org/10.1016/j.ajo.2005. 01.003.

11. Mees L, Upadhyaya S, Kumar P, Kotawala S, Haran S, Rajasekar $S$, et al. Validation of head- mounted virtual reality visual field screening device. J Glaucoma. 2020;29:86-91.

12. Moyer VA. and US preventive services task force. screening for glaucoma: U.S. preventive services task force recommendation statement. Ann Intern Med. 2013;159:484-9.

13. John D, Parikh R. Cost- effectiveness and cost utility of community screening for glaucoma in urban India. Public Health. 2017;148:37-48.

14. John D, Parikh R. Cost-effectiveness of community screening for glaucoma in rural India: a decision analytical model. Public Health. 2018;155:142-51.

15. Miller SE, Thapa S, Robin AL, Niziol LM, Ramulu PY, Woodward MA, et al. Glaucoma Screening in Nepal: cup to disc estimate with standard mydriatic fundus camera compared to portable non-mydriatic camera. Am J Ophthalmol. 2017;182: 99-106.

16. Sengupta S, Sindal MD, Besisrli CG, Upadhyaya S, Venkatesh R, Niziol LM, et al. Screening for vision-threatening diabetic retinopathy in South India:comparing portable non-mydriatic and standard fundus cameras and clinical exam. Eye. 2018;32:375-83.

17. Yogesan K, Constable IJ, Barry CJ, Eikelboom RH, Morgan W, Tay-Kearney ML, et al. Evaluation of a portable fundus camera for use in the teleophthalmologic diagnosis of glaucoma. J Glaucoma. 1999;8:297-301.

18. Munoz-Negrete FJ, Contreras I, Oblanca N, Pinazo-Duran MD, Rebolleda G. Diagnostic accuracy of non-mydriatic fundus photography for the detection of glaucoma in diabetic patients. Biomed Res Int. 2015;2015:1-8.

19. Detry-Morel M, Zeyen T, Kestelyn P, Collignon J, Goethals M. Belgian Glaucoma Society Screening for glaucoma in a general population with the non-mydriatic fundus camera and the frequency doubling perimeter. Eur J Ophthalmol.2004;14:387-93.

20. Tuulonen A, Airaksinen PJ, Montagna A, Nieminen H. Screening for glaucoma with a non-mydriatic fundus camera. Acta Ophthalmol. 1990;68:445-9.

21. Davila JR, Sengupta S, Niziol LM, Sindal MD, Besirli CG, Upadhyaya S, et al. Predictors of photographic quality with a handheld nonmydriatic fundus camera used for screening of vision-threatening diabetic retinopathy. Ophthalmologica. 2017; 238:89-99. 